\title{
Prevalence of cartilaginous tumours as an incidental finding on MRI of the knee
}

\author{
Wouter Stomp $^{1}$ • Monique Reijnierse ${ }^{1}$ - Margreet Kloppenburg ${ }^{2,3}$. \\ Renée de Mutsert ${ }^{3}$ - Judith V. M. G. Bovée ${ }^{4}$ Martin den Heijer ${ }^{3}$. \\ Johan L. Bloem ${ }^{1}$ • on behalf of the NEO study group
}

Received: 11 January 2015 /Revised: 25 March 2015 / Accepted: 2 April 2015 / Published online: 21 May 2015

(C) The Author(s) 2015. This article is published with open access at Springerlink.com

\begin{abstract}
Objectives The purpose was to determine prevalence of enchondromas and atypical cartilaginous tumour/ chondrosarcoma grade 1 (ACT/CS1) of the knee on MRI in a large cohort study, namely the Netherlands Epidemiology of Obesity (NEO) study.

Methods Participants aged 45 to 65 years were prospectively included, oversampling overweight and obese persons. Within a subgroup of participants, MRI of the right knee was performed and screened for incidental cartilaginous tumours, as defined by their characteristic location and appearance.

Results Forty-nine cartilaginous tumours were observed in 44 out of 1285 participants (estimated population prevalence $2.8 \%, 95 \%$ CI $2.0-4.0 \%$ ). Mean largest tumour diameter was $12 \mathrm{~mm}$ (range $2-31 \mathrm{~mm}$ ). Eight participants with a tumour larger than $20 \mathrm{~mm}$ or a tumour with aggressive features were referred to rule out low-grade chondrosarcoma. One was lost to follow-up, three had histologically proven ACT/CS1 and four had dynamic contrast MRI findings consistent with benign enchondroma.

Conclusions Incidental cartilaginous tumours were relatively common on knee MRI and may be regarded as a normal
\end{abstract}

Wouter Stomp

w.stomp@lumc.nl

1 Department of Radiology, C2-S, Leiden University Medical Center, P.O. Box 9600, 2300 RC Leiden, The Netherlands

2 Department of Rheumatology, Leiden University Medical Center, Leiden, The Netherlands

3 Department of Clinical Epidemiology, Leiden University Medical Center, Leiden, The Netherlands

4 Department of Pathology, Leiden University Medical Center, Leiden, The Netherlands concurrent finding. However, more tumours than expected were ACT/CS1. Because further examination was performed only when suspicion of chondrosarcoma was high, the actual prevalence might be even higher.

Key Points

- Incidental cartilaginous tumours are relatively common on knee MRI.

- Most incidental cartilaginous tumours are small and lack suspicious features.

- Small cartilaginous tumours without suspicious findings may be a normal concurrent finding.

- Large tumours and/or those with suspicious findings should be further investigated.

- Atypical cartilaginous tumour/chondrosarcoma grade 1 was found more often than expected.

Keywords Magnetic resonance imaging · Chondroma . Chondrosarcoma $\cdot$ Knee $\cdot$ Incidental findings

\section{Introduction}

Enchondromas are benign cartilaginous tumours located within the bone marrow. They are one of the most common osseous neoplasms and are often found incidentally on radiographs, computed tomography or magnetic resonance imaging (MRI), especially of the knee [1]. It is important to distinguish them from atypical cartilaginous tumour/ chondrosarcoma grade 1 (ACT/CS1), which requires surgical treatment. Differentiating them is difficult when they are located in the long tubular bones [2].

Enchondromas are composed of lobules of hyaline cartilage, consisting of benign chondrocytes with or without surrounding reactive bone formation [3]. Mutations of isocitrate dehydrogenase 1 (IDHI) and $I D H 2$ have been identified in 
enchondromas and chondrosarcomas, in patients with both solitary and multiple neoplasms $[4,5]$. Enchondromas are typically asymptomatic, except in the case of very large lesions which may be painful or may fracture.

During routine screening of knee MRIs for incidental findings, performed in a cohort study to investigate osteoarthritis, cartilaginous tumours were frequently observed. Because enchondromas are clinically silent, reliable data concerning their incidence and prevalence is scarce [1, $6]$. The purpose of this study was to describe the prevalence, characteristics and location of incidental cartilaginous tumours on knee MRI in a population-based cohort study.

\section{Materials and methods}

\section{Study design and study population}

The Netherlands Epidemiology of Obesity (NEO) study is a population-based prospective cohort study that includes 6673 individuals aged 45 to 65 years, with an oversampling of individuals deemed overweight or obese. This cohort was designed to prospectively study pathways that lead to disease in such individuals. Detailed information about the study design and data collection has been described elsewhere [7]. Men and women aged between 45 and 65 years with a self-reported body mass index (BMI) of $27 \mathrm{~kg} / \mathrm{m}^{2}$ or higher and living in the greater area of Leiden (in the west of the Netherlands) were eligible to participate in the NEO study. In addition, all inhabitants aged between 45 and 65 years from one nearby municipality (Leiderdorp) were invited, irrespective of their BMI. At baseline, participants completed a questionnaire about demographic and clinical data and underwent an extensive physical examination. In random subsamples of participants without contraindications, MRI of abdominal fat, pulse wave velocity of the aorta, heart, brain or right knee was performed. All MR images were screened for incidental findings. Incidental abnormal results with potential health consequences if left undiagnosed were disclosed to the participants and their general practitioners, accompanied by advice for further work-up.

The present study is a cross-sectional analysis of the baseline measurements of the 1285 participants with an MRI of the knee. The local medical ethics committee approved the study and all participants gave written informed consent.

All participants were asked whether they had pain in the right knee during most days of the previous month. Weight and height were measured without shoes and with precision of $0.1 \mathrm{~cm} / \mathrm{kg}$ and $1 \mathrm{~kg}$ was subtracted from the weight for clothing. BMI was calculated by dividing the weight in kilograms by the height in metres squared.

\section{MRI of the right knee}

MRI studies were performed on a 1.5-T MRI system (Philips, Best, the Netherlands) with a dedicated 8-channel knee coil. The following parameters were identical for the TSE images: echo train length 6, a 150-160 mm field of view and a $304 \times$ 512 matrix. Sequences acquired were (1) coronal proton density (PD) (repetition time (TR)/echo time (TE) 2335/35 ms; $3 \mathrm{~mm}$ slice thickness; $0.6 \mathrm{~mm}$ interslice gap); (2) coronal fat suppressed PD TSE images (TR/TE 2334/35 ms; 3 mm slice thickness; $0.6 \mathrm{~mm}$ interslice gap); (3) sagittal PD TSE images (TR/TE 2338/35; $3.5 \mathrm{~mm}$ slice thickness; $0.7 \mathrm{~mm}$ interslice gap); (4) sagittal frequency selective fat-suppressed T1weighted 3D gradient echo (GE) sequence (TR/TE 11/5.5; $25^{\circ}$ flip angle; $150 \mathrm{~mm}$ field of view, $272 \times 512$ matrix, $2 \mathrm{~mm}$ slice thickness with a $1-\mathrm{mm}$ overlap between images; no gap); (5) axial fat suppressed PD (TSE) images (TR/TE 3225/15; 4 mm slice thickness; $0.8 \mathrm{~mm}$ interslice gap). Total acquisition time was $30 \mathrm{~min}$.

\section{Lesion analysis}

A research fellow trained in reading knee MRIs initially screened all MRIs for the presence of any abnormality. All of these were subsequently discussed with a musculoskeletal radiologist. All lesions possibly fulfilling the criteria set for cartilaginous tumour were assessed by two musculoskeletal radiologists with more than 10 years experience in consensus to determine lesion characteristics. Cartilaginous tumours were defined as a smooth or lobulated lesion of geographic bone destruction pattern within the bone marrow with low signal on PD-weighted images and high signal on PD fatsuppressed images. Subchondral lesions were excluded, because they may represent subchondral cysts, intraosseous ganglia or subchondral oedema. We included and recorded characteristics of all other osseous lesions that could be bone tumours or tumour-like lesions of bone. For all included lesions we recorded size in three dimensions, shape (lobulated, oval or round), bone destruction pattern i.e. geographic with welldefined or partially ill-defined margins, permeative or motheaten. The location was determined to be either central or eccentric and located in the epiphysis, epi-metaphysis, metaphysis and/or diaphysis. The distance of the tumour to the growth plate was classified as a lesion being in contact with the physis, within $2 \mathrm{~cm}$, or further away from the physis. The relationship with the cortex in eccentric lesions was determined (contact or no contact). The presence or absence of superficial (one-third of depth) or deep (at least two-thirds of depth) scalloping (i.e. focal resorption of the inner margin of cortical bone), cortical bone destruction and periosteal reaction was recorded. Signal intensity was scored as low, intermediate or high compared to muscle on both PD and fatsuppressed PD-weighted images. The presence of speckled 
low signal intensity on both the PD and gradient-echo sequence in the lesion was scored as calcifications. Presence or absence of bone marrow oedema in direct contact or within $2 \mathrm{~cm}$ from the lesion was noted. Participants with lesions that were larger than $20 \mathrm{~mm}$ or that had aggressive characteristics (i.e. cortical bone destruction, periosteal reaction, deep endosteal scalloping, (partial) ill definition of margin) were contacted and referred for dynamic contrast MRI to differentiate enchondroma from ACT/CS1. Large lesion size is a known risk factor for chondrosarcoma but no specific limit is known which optimally differentiates between enchondroma and ACT/CS1. We chose a conservative cutoff of $20 \mathrm{~mm}$ as a trade-off between increased sensitivity and avoiding unnecessary extra diagnostic procedures.

\section{Dynamic contrast MR}

Data acquisition was performed during intravenous injection of Gd-DTPA (dose $2 \mathrm{mg} / \mathrm{kg}$ body weight, power injector with $2 \mathrm{ml} / \mathrm{s}$ ) with a temporal resolution of $3 \mathrm{~s}$. We recorded on electronic subtraction images the time interval between arterial enhancement and the start of lesion enhancement. Lesions enhancing within $10 \mathrm{~s}$ were classified as early enhancement consistent with ACT/CS1, while lesions that did not enhance, or enhanced after more than $10 \mathrm{~s}$ were classified as enchondroma [8]. In addition we retrieved follow-up radiographs, and MRI studies when these had been performed. Changes of described parameters between initial and followup MRI studies and radiographs were recorded.

\section{Histological diagnosis}

When the aforementioned features on radiographs or MRI suggesting the presence of $\mathrm{ACT} / \mathrm{CS} 1$ were present, material was obtained (curettage or resection) to allow a histological diagnosis to be made. Previously described histological criteria were used [9]. All involved pathologists had extensive experience with bone tumour pathology owing to the hospital's function as a tertiary referral centre for orthopaedic oncology.

\section{Statistical analyses}

Characteristics of the lesions were expressed as number of the total number of lesions. In order to correctly estimate the population prevalence of cartilaginous tumours and represent associations in the general population, all other results were based on weighted analyses adjusting for the oversampling of persons with a BMI of $27 \mathrm{~kg} / \mathrm{m}^{2}$ or higher in the NEO study [10]. This was done by weighing individuals towards the BMI distribution of participants from the Leiderdorp municipality [11], whose BMI distribution was similar to the BMI distribution of the general Dutch population [12]. Weighted baseline characteristics of the study population were expressed as mean (SD) or as percentage. Participants were categorized into four groups based on their BMI $(\mathrm{BMI}<25,25 \leq \mathrm{BMI}<30,30 \leq$ $\mathrm{BMI}<40$ and $\mathrm{BMI} \geq 40 \mathrm{~kg} / \mathrm{m}^{2}$ ). Logistic regression analysis was used to examine the associations of BMI (both as a continuous variable and as a categorical variable using $\mathrm{BMI}<$ $25 \mathrm{~kg} / \mathrm{m}^{2}$ as the reference group), age (continuous), sex, and knee pain with the presence of cartilaginous tumours.

\section{Results}

Between September 2008 and October 2012, 6673 persons aged 45 to 65 years were included in the NEO study, of whom 1285 underwent MRI of the right knee. Weighted mean (SD) age was 56 (6) years, $45 \%$ were men and mean BMI was 27.1 (4.7) $\mathrm{kg} / \mathrm{m}^{2}$. After screening of these 1285 MRIs, 49 lesions in 44 participants were observed and classified as cartilaginous tumours (estimated population prevalence $2.8 \%, 95 \% \mathrm{CI}$ $2.0-4.0 \%$ ). Four participants had more than one lesion: three participants had two and one participant had three lesions. Using logistic regression analysis, we did not find any association between BMI, age or sex and the presence of
Fig. 1 Small enchondroma in the distal femur located centrally in the metaphysis and with a typical lobulated appearance: a PDweighted coronal section, b PDweighted coronal section with fat saturation. No further imaging was obtained
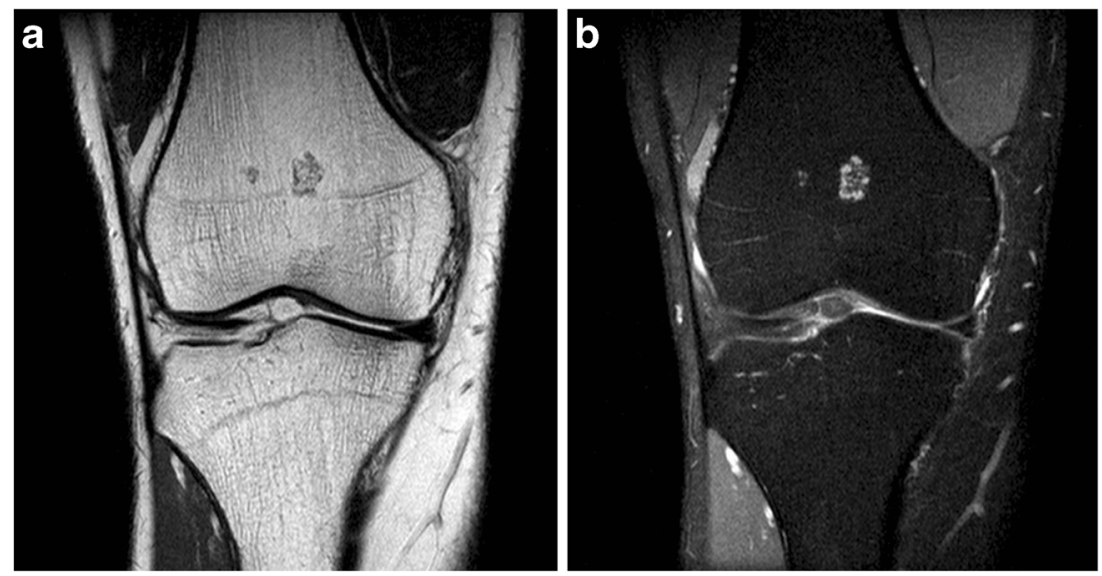
cartilaginous tumours (data not shown). An example of a typical enchondroma is shown in Fig. 1. We did not encounter any non-cartilaginous bone tumours.

\section{Lesion characteristics}

Mean lesion size was 12 (range $2-31$ ) $\mathrm{mm}$ in the longest axis. Of the 49 tumours, seven tumours were larger than $20 \mathrm{~mm}$. Thirty-six lesions were lobulated, 12 had a smooth round shape and one was oval-shaped. All had a geographic bone destruction pattern and were well defined. Thirty-eight were located in the distal femur, eight in the proximal tibia, two in the proximal fibula and one in the patella. Nineteen tumours were located centrally in the medullary canal and 30 eccentrically (but still within the medullary canal). Of all tumours, 27 were located in the metaphysis, 10 in the epi-metaphysis (crossing the growth plate), ten in the diaphysis and one in the epiphysis (not applicable for the lesion in the patella). Twenty-nine were in contact with the growth plate, nine crossed the growth plate and ten were located more than $2 \mathrm{~cm}$ from the growth plate (not applicable for the
Fig. 2 Large cartilaginous tumour centrally located in the distal femur, referred for followup: a axial and b sagittal T1weighted images, $\mathbf{c}$ axial T2weighted image, $\mathbf{d}$ axial and $\mathbf{e}$ sagittal T1-weighted images after gadolinium administration with fat suppression. The time-signal intensity curve of the dynamic MRI (e) showed slow

enhancement consistent with enchondroma (pink artery, orange tumour, and blue bone marrow as reference tissue). The vertical axis represents relative signal intensity and the horizontal axis represents time in seconds

lesion in the patella). In 11 lesions there was contact with the cortex and in one lesion there was focal destruction of the cortex. In three cases there was minimal and in one case obvious deep endosteal scalloping. Periosteal reaction was not seen. Signal intensity was intermediate on PD images and heterogeneously high on PD-SPIR images for all lesions. The presence of calcifications within the lesion was observed in 11 lesions. None of the lesions had surrounding oedema.

Eight lesions in eight participants displayed at least one of the predefined aggressive criteria and were referred for follow-up. Seven had a tumour larger than $20 \mathrm{~mm}$ (range $22-31 \mathrm{~mm}$ ), including one with deep endosteal scalloping. One participant had a smaller tumour but with focal cortical destruction. In six of the seven participants referred because of tumour size, dynamic contrast MRI was performed, while one patient was subsequently lost to follow-up. In four participants there was slow lesional enhancement (i.e. the interval between arterial enhancement and lesion enhancement was greater than $10 \mathrm{~s}$ ) after intravenous Gd-chelate administration consistent with benign enchondroma (Fig. 2); no subsequent follow-up was performed in these patients. In the fifth patient with a lesion of $22 \mathrm{~mm}$, and in the sixth patient with a larger lesion with deep endosteal scalloping, dynamic contrast MRI demonstrated a fast enhancement pattern consistent with ACT/CS1. Curettage and en bloc resection of the tumour were performed respectively, and pathology confirmed the diagnosis of ACT/CS1 (Fig. 3). The patient with the smaller lesion but with focal cortical destruction had radiographic follow-up and underwent curettage 2 years later, showing an ACT/CS1 at histology. The three ACT/ $\mathrm{CS} 1 \mathrm{~s}$ that were found correspond to an estimated population prevalence of $0.4 \%$ (95\% CI $0.2-0.8 \%)$.

\section{Association of BMI, age, sex and knee pain with presence of cartilaginous tumours}

Estimated population prevalence of cartilaginous tumours in BMI strata are shown in Table 1. Univariate logistic regression analysis did not reveal associations between BMI, age, sex and the presence of cartilaginous tumour, but it was suggestive of an inverse association with knee pain (Table 2). Multivariate logistic regression using the same variables showed an inverse association between the presence of knee pain and the presence of cartilaginous tumour but no association with BMI (as continuous variable), age or sex. Compared with having a BMI $<25 \mathrm{~kg} /$ $\mathrm{m}^{2}$, the odds ratio (OR, $95 \%$ confidence interval) of the presence of cartilaginous tumour associated with a BMI of $25-30 \mathrm{~kg} / \mathrm{m}^{2}$ was $1.79(0.38,8.51)$, with a BMI of 30
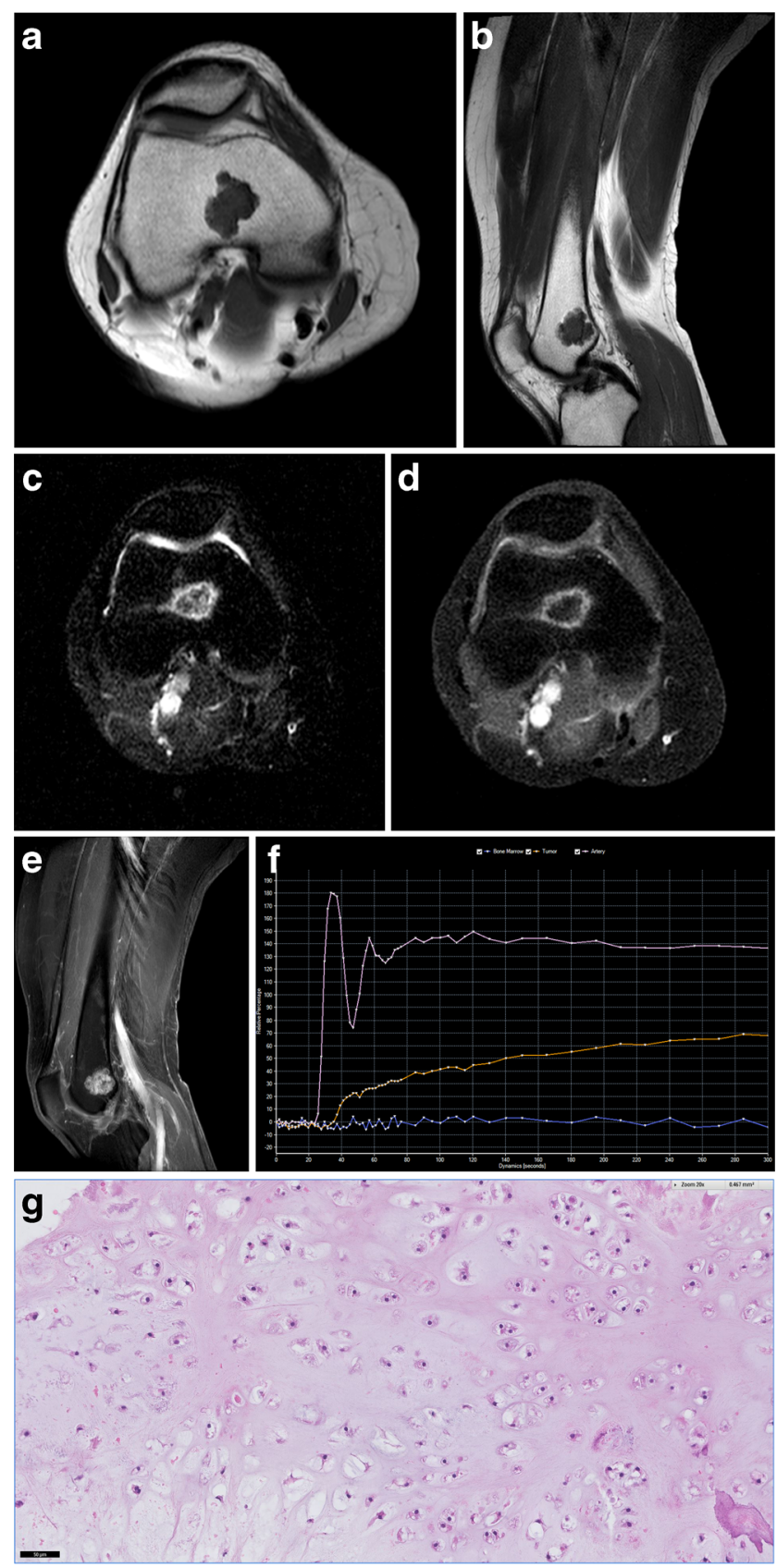

Fig. 3 Large cartilaginous tumour centrally located in the distal femur, referred for follow-up: a axial and $\mathbf{b}$ sagittal T1-weighted images, $\mathbf{c}$ axial T2-weighted image, d axial and e sagittal T1-weighted images after gadolinium administration with fat suppression. The time-signal intensity curve of the dynamic MRI (e) showed fast enhancement $(<10 \mathrm{~s})$, interpreted as most likely ACT/CS1, although chondrosarcoma grade 2 could not be excluded due to extensive presence of mucoid (pink artery, orange tumour, and blue bone marrow as reference tissue). The vertical axis represents relative signal intensity and the horizontal axis represents time in seconds. Curettage was performed and histology (f) confirmed the presence of cartilaginous tumour with increased cellularity and occasional binucleated cells as well as focal mucomyxoid matrix changes (left lower area), diagnosed as atypical cartilaginous tumour/ chondrosarcoma grade 1

$40 \mathrm{~kg} / \mathrm{m}^{2}$ it was $1.69(0.38,7.47)$, and with a BMI $>40 \mathrm{~kg} /$ $\mathrm{m}^{2}$ it was $3.74(0.64,21.87)$. 
Table 1 Participant

characteristics and prevalence of cartilaginous tumours by BMI groups

\begin{tabular}{|c|c|c|c|c|}
\hline & \multicolumn{4}{|c|}{ BMI $\left(\mathrm{kg} / \mathrm{m}^{2}\right)$} \\
\hline & $\begin{array}{l}<25 \\
(35 \%)\end{array}$ & $\begin{array}{l}25-30 \\
(44 \%)\end{array}$ & $\begin{array}{l}30-40 \\
(19 \%)\end{array}$ & $\begin{array}{l}\geq 40 \\
(2 \%)\end{array}$ \\
\hline Age (years) & $56(6)$ & $56(6)$ & $55(6)$ & $53(6)$ \\
\hline Sex (\% women) & 63 & 46 & 60 & 84 \\
\hline BMI $\left(\mathrm{kg} / \mathrm{m}^{2}\right)$ & $22.6(1.5)$ & $27.4(1.3)$ & $33.3(2.7)$ & $42.9(2.4)$ \\
\hline Cartilaginous tumour prevalence $(\%)$ & 1.9 & 3.2 & 3.1 & 7.1 \\
\hline Knee pain (\% yes) & 14 & 17 & 23 & 27 \\
\hline
\end{tabular}

Results are based on weighted analysis of the study population $(n=1285)$. Values are presented as mean (SD) or percentages

\section{Discussion}

In a cohort study including knee MRI to investigate osteoarthritis, we observed incidental lesions that we classified as cartilaginous tumours on the basis of predefined imaging criteria in $2.8 \%$ of individuals between 45 and 65 years of age. These cartilaginous tumours were all smaller than $32 \mathrm{~mm}$, typically smaller than $20 \mathrm{~mm}$ and were located near the femoral or sometimes tibial metaphysis.

The prevalence of ACT/CS1 in our population was low $(0.4 \%)$, but we found an ACT/CS1 in three out of seven participants whom we classified, on the basis of predefined criteria, as high risk of carrying an ACT/CS1 and had a complete data set. These ACT/CS1s were present in two participants with tumours exhibiting aggressive features (deep endosteal scalloping or cortical bone destruction) and one participant with a tumour of $22 \mathrm{~mm}$ and were either resected or removed by curettage. In four other lesions with a diameter of greater than $20 \mathrm{~mm}$ the criteria of dynamic MRI were consistent with an indolent benign enchondroma [8].

Because cartilaginous tumours are typically clinically silent reliable data concerning their epidemiology are scarce. Similar to our study, Walden et al. reported an enchondroma prevalence of $2.9 \%$ on clinical knee MRI [13]. In skeletally immature children undergoing knee MRI for a variety of

Table 2 Univariate and multivariate analysis for risk factors of presence of cartilaginous tumours

\begin{tabular}{llllll}
\hline Variables & \multicolumn{2}{l}{ Univariate analysis } & & \multicolumn{2}{l}{ Multivariate analysis } \\
\cline { 2 - 3 } \cline { 6 - 7 } \cline { 6 - 7 } & OR $(95 \% \mathrm{CI})$ & $P$ & & OR $(95 \% \mathrm{CI})$ & $P$ \\
\hline BMI $\left(\mathrm{kg} / \mathrm{m}^{2}\right)$ & $1.05(0.97-1.14)$ & 0.202 & & $1.06(0.98-1.14)$ & 0.168 \\
Age (year) & $0.97(0.91-1.04)$ & 0.400 & & $0.98(0.91-1.05)$ & 0.482 \\
Sex (woman) & $1.20(0.45-3.14)$ & 0.717 & & $1.23(0.47-3.23)$ & 0.673 \\
Knee pain (yes) & $0.44(0.18-1.10)$ & 0.080 & & $0.40(0.18-0.93)$ & 0.034 \\
\hline
\end{tabular}

Results are based on weighted analysis of the study population $(n=1285)$

$C I$ confidence interval, $O R$ odds ratio indications, enchondroma prevalence was also $2.9 \%$ [14]. In neither of these two studies was further follow-up performed. Our study confirms these results with a similar prevalence of cartilaginous tumours of $2.8 \%$. However, we also showed that ACT/CS1s are relatively common among larger tumours and those with suspicious features. In an autopsy case series, a prevalence of enchondromas of only $0.2 \%$ was observed, likely reflecting the higher sensitivity of MRI for detecting small lesions [15]. Other asymptomatic osseous tumours were not encountered in our cohort, which means that the prevalence of these is, in our age group, less than that of cartilaginous tumours and below $0.1 \%$. Our data did not reveal a clear association with age, sex or BMI as a continuous variable, and an inverse association with knee pain. The latter finding may merely reflect that participants with cartilaginous tumours do not suffer from knee pain. There was a clear trend towards a higher prevalence in higher BMI groups; however, the number of detected cartilaginous tumours was probably too low to reach a significant association in the logistic regression analysis.

The high sensitivity of MRI in detecting small cartilaginous tumours on MRI performed for other purposes can pose a dilemma to the clinician. The main differential diagnosis of enchondroma is ACT/CS1. In high-grade chondrosarcoma, clinical, radiographic and pathological findings generally are apparent and treatment consists of surgical resection with a wide margin. However, differentiating ACT/CS1 from enchondroma is challenging, especially in the case of incidental asymptomatic lesions found on imaging. Asymptomatic enchondromas do not need treatment, while ACT/CS1s are treated surgically [16].

Suggestions on how to address this dilemma have been made (Table 3). Radiographic signs are not useful in further classifying these small enchondromas. In a previous radiograph-based case series, location in the axial skeleton and size greater than $5 \mathrm{~cm}$ were the most reliable predictors for low-grade chondrosarcoma, while clinical symptoms and morphologic radiographic features did not have added value [17]. Radiographic signs indicating potential 
Table 3 Imaging features reported in literature to be suggestive of chondrosarcoma

Pain related to the tumour

Location in the axial skeleton

Large lesion size $(>5 \mathrm{~cm})$

Lobulated contour

Ill-defined margin

Enlargement of the medullary cavity and cortical thickening

Endosteal scalloping $>2 / 3$ of the cortical thickness or over $>2 / 3$ of the lesion length

Neurovascular involvement

Liquefaction

Peritumoral oedema*

Moth-eaten or permeative osteolysis*

Spontaneous pathologic fracture*

Periosteal reaction*

Cortical destruction*

Soft-tissue extension*

Early $(<10 \mathrm{~s})$ enhancement on dynamic contrast MRI

Intense uptake on bone scintigraphy

FDG-PET high uptake*

Imaging features reported in the literature making a diagnosis of chondrosarcoma more likely. List synthesized from refs. [17-21]

*Features suggestive of high-grade (grade 1 and 2) chondrosarcoma

chondrosarcoma have been described by Parlier-Cuau et al.; however, no radiographs were made in our cohort [18]. Only bone scintigraphy (intense uptake on delayed images without presence of fracture) and dynamic Gdchelate-enhanced MRI (start of enhancement within $10 \mathrm{~s}$ after arterial enhancement) have been reported to add information on differentiating enchondroma from ACT/CS1 [19-21]. We observed MRI signs suggestive of chondrosarcoma such as deep endosteal scalloping, cortical destruction and soft-tissue extension in two out of three participants who indeed turned out to have ACT/ CS1 [21]. Nevertheless, even with dynamic contrastenhanced MRI, differentiating cartilaginous tumours remains challenging with false-positive findings unavoidable [22].

Chondrosarcoma incidence has been estimated at $0.0005 \%$ per year and prevalence at $0.001-0.009 \%$, with only $7 \%$ of those located around the knee [23-25]. In our study we observed an unexpectedly high estimated population prevalence of $\mathrm{ACT} / \mathrm{CS} 1$ of $0.4 \%$. As no further examinations or follow-up were performed when tumours were small and lacked suspicious features, the actual prevalence of ACT/CS1 may be even higher. Thus knee MRI performed in participants should be carefully reviewed for the presence of cartilaginous tumours. Only small lesions lacking suspicious features may be regarded as a normal concurrent finding on MRI. In patients with any of the described suspicious features, the chance of having an ATC/CS1 is quite high (three out of seven in our population).

\section{Limitations}

The present study was conducted in a cohort study of volunteers. Compared to the general population, participants may be more conscious about their health. As a consequence, some may have had a healthier lifestyle, while others may have had more health complaints and participated because of the diagnostic examinations performed as part of the study. Furthermore, a large proportion of participants were overweight or obese. To estimate the population prevalence we weighted the analyses towards the BMI distribution of a population comparable to the general Dutch population. Radiographs were not available for the study group, making it impossible to determine the discrepancy between prevalence on radiographs and MRI. However, because the estimated population prevalence in our study was similar to that in earlier studies, we are confident that our results are robust.

Because of the benign nature of enchondromas, there are no histopathological studies confirming the origin of the otherwise unsuspicious lesions and they are classified as enchondromas solely on imaging findings. As we did not routinely do resections and do not yet have 10-year followup, the proof of excluding ACT/CS1 in the three unsuspicious larger lesions was incomplete.

\section{Conclusion}

The estimated population prevalence of incidental asymptomatic cartilaginous tumours around the knee was $2.8 \%$. Typically the lesions were less than $20 \mathrm{~mm}$ in size, were located within $2 \mathrm{~cm}$ of the growth plate and did not cause cortical abnormalities. The prevalence of ACT/CS1 may be higher than previously thought $(0.4 \%)$. Given the high prevalence of enchondromas, as a practical rule we do not advocate further imaging examination or follow-up of accidentally found cartilaginous tumours in patients up to 65 years when the tumours are small (at most $20 \mathrm{~mm}$ ) and suspicious features such as deep endosteal scalloping or cortical destruction are absent. However, when any of these suspicious features are present, or when the lesion is larger than $20 \mathrm{~mm}$ in diameter, further analysis is indicated.

Acknowledgments The scientific guarantor of this publication is J.L. Bloem. The authors of this manuscript declare no relationships with any companies whose products or services may be related to the subject matter of the article. The NEO study is supported by the participating Departments, the Division and the Board of Directors of the Leiden University Medical Centre, and by the Leiden University Research Profile Area 'Vascular and Regenerative Medicine'. One of the authors (RdM) has 
significant statistical expertise and was involved with or performed statistical analyses. Institutional review board approval was obtained. Written informed consent was obtained from all subjects in this study. The study design was published previously (Eur J Epidemiol. 2013 Jun;28(6):513-23) and several reports have been published on the study subjects unrelated to the current study topic. Methodology: prospective cross sectional study performed at one institution.

We would like to thank O.M. Dekkers, S. Middeldorp and P.R. Kornaat for their involvement in screening of the knee MRIs for incidental findings and further management of these findings. We also express our gratitude to all individuals who participated in the Netherlands Epidemiology in Obesity study, and we are grateful to all participating general practitioners for inviting eligible participants. We furthermore thank all research nurses for collecting the data and I. de Jonge, MSc for all data management of the NEO study.

This report was written on behalf of the NEO study group, further consisting of Frits R. Rosendaal, Renée de Mutsert, Ton J. Rabelink, Johannes W.A. Smit, J. Wouter Jukema, Albert de Roos, Saskia le Cessie, Pieter S. Hiemstra, Margreet Kloppenburg, Tom W.J. Huizinga, Hanno Pijl, Eelco J.P. de Koning, Willem J.J. Assendelft, Pieter H. Reitsma, Ko Willems van Dijk, Aiko P.J. de Vries, Hildo J. Lamb, Ingrid M. Jazet, Olaf M. Dekkers, Nienke R. Biermasz, Jeanet W. Blom, Patrick C.N. Rensen, Christa M. Cobbaert (Leiden University and Medical Center, Leiden, the Netherlands), Martin den Heijer, Jacqueline M. Dekker and Brenda W. Penninx (VU Medical Center, Amsterdam, the Netherlands).

Open Access This article is distributed under the terms of the Creative Commons Attribution-NonCommercial 4.0 International License (http:// creativecommons.org/licenses/by-nc/4.0/), which permits any noncommercial use, distribution, and reproduction in any medium, provided you give appropriate credit to the original author(s) and the source, provide a link to the Creative Commons license, and indicate if changes were made.

\section{References}

1. Kransdorf MJ, Peterson JJ, Bancroft LW (2007) MR imaging of the knee: incidental osseous lesions. Magn Reson Imaging Clin N Am 15:13-24

2. Flemming DJ, Murphey MD (2000) Enchondroma and chondrosarcoma. Semin Musculoskelet Radiol 4:59-71

3. Milgram JW (1983) The origins of osteochondromas and enchondromas. A histopathologic study. Clin Orthop Relat Res 174:264-284

4. Amary MF, Bacsi K, Maggiani $F$ et al (2011) IDH1 and IDH2 mutations are frequent events in central chondrosarcoma and central and periosteal chondromas but not in other mesenchymal tumours. J Pathol 224:334-343

5. Pansuriya TC, van Eijk R, d' Adamo P et al (2011) Somatic mosaic IDH1 and IDH2 mutations are associated with enchondroma and spindle cell hemangioma in Ollier disease and Maffucci syndrome. Nat Genet 43:1256-1261

6. Douis H, Saifuddin A (2012) The imaging of cartilaginous bone tumours. I. Benign lesions. Skelet Radiol 41:1195-1212

7. De Mutsert R, den Heijer M, Rabelink TJ et al (2013) The Netherlands Epidemiology of Obesity (NEO) study: study design and data collection. Eur J Epidemiol 28:513-523
8. Geirnaerdt MJ, Hogendoorn PC, Bloem JL et al (2000) Cartilaginous tumors: fast contrast-enhanced MR imaging. Radiology 214:539-546

9. Hogendoorn PCW, Bovée JVMG, Nielsen GP (2013) Chondrosarcoma (grades I-III), including primary and secondary variants and periosteal chondrosarcoma. In: Fletcher CDM, Bridge JA, Hogendoorn PCW, Mertens F (eds) World Health Organization classification of tumours. Pathology and genetics of tumours of soft tissue and bone, 4th edn. International Agency for Research on Cancer, Lyon, pp 264-268

10. Korn EL, Graubard BI (1991) Epidemiologic studies utilizing surveys: accounting for the sampling design. Am J Public Health 81: $1166-1173$

11. Lumley T (2004) Analysis of complex survey samples. J Stat Softw 9:1-19

12. Ministerie van VWS (2012) Hoeveel mensen hebben overgewicht? National Institute for Public Health and the Environment (RIVM). http://www.rivm.nl/dsresource?objectid=rivmp:76024\&type= org\&disposition=inline\&ns_nc=1. Accessed Aug 2013

13. Walden MJ, Murphey MD, Vidal JA (2008) Incidental enchondromas of the knee. AJR Am J Roentgenol 190:1611-1615

14. Douis H, Davies AM, James SL et al (2012) Can MR imaging challenge the commonly accepted theory of the pathogenesis of solitary enchondroma of long bone? Skelet Radiol 41:1537-1542

15. Scherer E (1928) Exostosen, Enchondrome und ihre Beziehung zum Periost. Frankfurt Ztschr f Path 36:587-605

16. Ryzewicz M, Manaster BJ, Naar E, Lindeque B (2007) Low-grade cartilage tumors: diagnosis and treatment. Orthopedics 30:35-46

17. Geirnaerdt MJ, Hermans J, Bloem JL et al (1997) Usefulness of radiography in differentiating enchondroma from central grade 1 chondrosarcoma. AJR Am J Roentgenol 169:1097-1104

18. Parlier-Cuau C, Bousson V, Ogilvie CM et al (2011) When should we biopsy a solitary central cartilaginous tumor of long bones? Literature review and management proposal. Eur J Radiol 77:6-12

19. Lee FY-I, Yu J, Chang S-S et al (2004) Diagnostic value and limitations of fluorine-18 fluorodeoxyglucose positron emission tomography for cartilaginous tumors of bone. J Bone Joint Surg Am 86-A:2677-2685

20. Van Rijswijk CSP, Geirnaerdt MJA, Hogendoorn PCW et al (2004) Soft-tissue tumors: value of static and dynamic gadopentetate dimeglumine-enhanced MR imaging in prediction of malignancy. Radiology 233:493-502

21. Murphey MD, Flemming DJ, Boyea SR et al (1998) Enchondroma versus chondrosarcoma in the appendicular skeleton: differentiating features. Radiographics 18:1213-1237

22. De Coninck T, Jans L, Sys G et al (2013) Dynamic contrastenhanced MR imaging for differentiation between enchondroma and chondrosarcoma. Eur Radiol 23:3140-3152

23. Giuffrida AY, Burgueno JE, Koniaris LG et al (2009) Chondrosarcoma in the United States (1973 to 2003): an analysis of 2890 cases from the SEER database. J Bone Joint Surg Am 91: $1063-1072$

24. Orphanet (2103) Chondrosarcoma. http://www.orpha.net/consor/ cgi-bin/OC_Exp.php?Expert=55880. Accessed 26 Nov 2013

25. Yochum TR, Rowe LJ (2004) Essentials of skeletal radiology, 3rd edn. Lippincott Williams \& Wilkins, Baltimore 